
\title{
$\begin{array}{ll}\text { Research Square } & \text { They should not be considered conclusive, used to inform clinical practice, } \\ \text { or referenced by the media as validated information. }\end{array}$
}

\section{RootlX: An Easy and Ready to Use Tool for the Study of Plant Root Growth and Architecture.}

Maxime Gotté

Université de Rouen https://orcid.org/0000-0001-7192-0945

Quentin Amaudin

Universite de Rouen UFR des Sciences et Techniques

Gaëlle Durambur

Universite de Rouen

Yohana Laloum

Universite de Rouen UFR des Sciences et Techniques

Azeddine Driouich

Universite de Rouen UFR des Sciences et Techniques

Marie-Laure Follet-Gueye

Universite de Rouen UFR des Sciences et Techniques

Eric Nguema-Ona

Groupe Roullier CMI

Maité Vicré-Gibouin ( $\nabla$ maite.vicre@univ-rouen.fr)

Normandie Université, SFR Normandie Végétal, Université de Rouen, Laboratoire Glyco-MEV EA43581.

\section{Software}

Keywords: Excel, root architecture, visual basic, root growth, root length, lateral root formation

Posted Date: November 5th, 2020

DOI: https://doi.org/10.21203/rs.3.rs-101192/v1

License: @) (7) This work is licensed under a Creative Commons Attribution 4.0 International License. Read Full License 


\section{Abstract}

Background: Agronomical and plant physiological studies employ a large variety of compounds that impact root development. The effect of these compounds is often evaluated by root architecture analyses using dedicated software. During the past decade, a growing number of tools proposed complex and deep analysis of the root system architecture. While these software applications are often complex and require specific set up, here, we propose a simple method based on the most common tools used by biologists: ImageJ and Excel®.

Results: First, roots are measured manually with ImageJ following a succession of operations ( $i$. choose a plant; $i i$. Measure: $a$. the primary root - $b$. lateral roots). Secondly, RootIX, an Excel file with dedicated macros that will automatically extract, sort and treat the data, is launched. Tables and graphics describing the data are generated. Root length, elongation rate and additional lateral roots are the main indicators provided among many others. We evaluated the effect of algae extracts on tomato roots growth with RootIX. We highlighted that these compounds negatively affect root growth while promoting generation of lateral roots.

Conclusion: Ease of use and settings as well as complete data analyses without any cost are the scaffold of RootIX.

\section{Background}

Growing in sand, mud, rocks and water, the sessile plants expand their roots to find nutrients and water. During their deployment roots will be challenged many times when microbes would try to invade their tissues (Chuberre et al. 2018) as well as dehydration or a changing salt concentration would generate osmotic stress (Robbins and Dinneny 2015).

Thus, this organ may extend and modulate its growth to ensure the best supply of water and nutrients to the whole plant (Robbins and Dinneny 2015). The best way to efficiently carry out these functions is to optimize the volume of soil explored by the root and optimize the root surface area (Satbhai et al. 2015). These parameters are significantly defined by the 3D configuration of the root system architecture. Indeed the spatial organization (root sizes, number and distribution of lateral roots) and the length and density of root hairs may affect drastically the soil/root interface surface (Satbhai et al. 2015).

The root behavior is controlled by intrinsic pathways that refer to the plant internal conditions such as hormonal balance and their receptor availability, transcription factors activation; and extrinsic pathways where the similar networks are monitored by environmental stimuli such as soil moisture or pathogens aggression (Jung and McCouch, 2013). Thus the perception of a microbe associated molecular patterns (MAMP) such as the bacterial flagellin peptide (flg22) affects the root growth and lateral root formation of Arabidopsis thaliana (Beck et al. 2014; Stringlis et al. 2018).

Control of root growth is mediated by a complex balance between hormones. This implies antagonism, e.g. auxin promotes lateral root initiation (Dubrovsky et al. 2008) while it is inhibited by gibberellins (Gou et al. 2010); and interaction, e.g. jasmonates promote lateral root formation through its interaction with auxin but also inhibit primary root growth (Xue and Zhang 2007; Sun et al. 2009). To gain more details on the subtle hormone control of root growth, the reader may appreciate the nice reviews written by Jung and McCouch, 2013 or Rutten and ten Tusscher, 2019.

Despite the strong interest to understand how the hormonal balance may be controlled by a given treatment, in many cases, the point to determine is just the effect on elongation and lateral root formation.

Numerous software solutions bring extensive and deep root architectural analysis, such as WinRHIZO, IJ_Rhizo (Pierret et al. 2013) or archiDART (Delory et al. 2018). However, many scientists and papers prefer to measure root lengths with their regular tools (ImageJ, ruler,...) (Li et al. 2014; Pereira and Pereira 2018; Fonouni-Farde et al. 2019) rather than investing time and money in a strong and often complex root architecture analysis software.

We faced this context while studying the effect of algae extracts on roots. After several failures with some of the above-mentioned programs, we get back to ImageJ manual measurements and developed the RootIX solution to extract, sort and treat the huge quantity of generated data.

Finally, we propose to share this tool that may be appreciated by all of those who just need the essential details about root growth and lateral root formation rather than complete $3 \mathrm{D}$ architecture, and by the way who want to save time and money.

\section{Implementation}

\section{Plant materials and growth conditions}

Although this method and tool can be used with many plant and root types, we developed it on tomato (Solanum lycopersicum var. St Pierre) seedlings that were used for all the experiments.

Seeds were surface sterilized with $70 \%(\mathrm{v} / \mathrm{v})$ ethanol for $1 \mathrm{~min}$ followed by immersion in $0.87 \%(\mathrm{v} / \mathrm{v})$ bleach for 1 min. After several washes in sterile distilled water, the seeds were stratified five days at $4^{\circ} \mathrm{C}$ to promote seed germination and finally sown on half-strength MS medium (Sigma). Agar $1 \%$ (Sigma) was used as a gelling agent. Media is disposed in $24 \mathrm{~cm} \times 24 \mathrm{~cm}$ plates (Thermo Scientific ${ }^{\mathrm{TM}}$ Nunc ${ }^{\mathrm{TM}}$ Square BioAssay Dishes $500 \mathrm{~cm}^{2}$ ). Seeds were grown in a photoperiodic chamber under an $16 \mathrm{~h}$ daylight period at $25^{\circ} \mathrm{C}$ for ten days before treatment. $19.2+/-2.4$ plants were analyzed per plates.

Each seedling was subjected to different treatments (one treatment per plate). Autoclaved mineral water (Cristalline® Emma source) was used as solvent and negative control. The bacterial peptide flagelline 22 (flg22) was used at $1 \mu M$ (Millet et al. 2010; Plancot et al. 2013). Two algal extracts produced by the CMI (Roullier), referred to as AlgEx1 and AlgEx2, were diluted before use at three total carbon concentrations (ppm) AlgEx1: 2000, 6000 and 8000; AlgEx2: 1000, 4000 and 8000 . 


\section{Computing environment}

RootIX is an Excel file with a batch of macros developed in Visual Basic for Excel which is the well-known spreadsheet developed by Microsoft. This commonly used software is part of the Microsoft Office 365 Suit available on https://products.office.com for Windows and MACOS. Notice that it may be free for university members. Furthermore, RootlX is compatible with all Excel version since the 2007 version until the Microsoft Office 365 suit.

A macro is an automated program that can be launch from a simple button present in an Excel worksheet. Here, RootIX will analyze and organize the data obtained with ImageJ.

Image J is a public image processing program developed at the National Institute of Health. It allows to analyze images with a large variety of tools. Image J can be downloaded for free from http://rsbweb.nih.gov/ij/ and is available for Windows, Mac OS and Linux. Although ImageJ's potential is almost unlimited (Schindelin et al. 2015), here we only require the simplest tool of "Segmented Line" measurement (right click on the line symbol).

\section{Images acquisition}

There is no precise images properties requirement if their quality is good enough to enable precise measurement and allow to distinguish roots that cross each other.

Here, images of plant were acquired with a scanner (Epson Expression 11000XL). Since the scans were conducted on sealed plates, the focus was manually adjusted to 5.0 in order to reach the best focus. Scan images were saved in ".tif" with the following routine properties: $800 \mathrm{dpi}$, 48 -bit color depth.

\section{Root sequential measurements}

Roots are measured with the ImageJ segmented line tool. To get the appropriate length values, make sure to adjust the scale.

It is required to conduct the measurements in a specific sequence (Fig1):

1. Choose a plant

2. Measure the primary root length (See the "special cases" section below, for monocotyledons or indiscernible primary root).

3. From the primary root apex, go back up to the hypocotyl measuring each lateral root on the way.

*Triple the record of a measurement for tertiary roots.

*Quaternary roots or higher order of ramification are not taken into account and should be recorded as tertiary roots.

4. Choose a second plant and follow the same sequence.

5. When the measurements of the picture are done, save the measurement results in ".tsv" (preferentially) or ".csv" file format. It is required to give a name that contains the condition (e.g. "water"), the time point (e.g. "t01h") and the replicate (e.g. "R1"). In the examples given above the file name would be: "water-t01h-R1.tsv". See other examples in the "legend and examples" sheet of the RootIX file.

It is recommended to use at least five decimals on the ImageJ records (on ImageJ: Analyze - set measurements... - decimal places (0-9): 5).

\section{Data analysis}

\section{a. How to launch the analysis:}

Data analysis can be launched in 3 simple steps.

1. Fill in the "Parameters" sheet required cells (pale green cells) with their appropriate information following the instructions in red cells:

a. File path in "B1"to access the folder that contain the data files.

b. A giving name of the experiment in "B2".

c. Primary and lateral roots discrimination size in "E2" (primary root (RI) will be considered as higher and lateral root as lower than this size). See the "special cases" section below for size lower than $1 \mathrm{~cm}$ and lateral roots longer than RI.

d. Data name information of each file to analyze ("conditions" row 7 and when it is suitable "replicate", "starting" and "ending time point" respectively rows 8,9 and 10). This batch of information is required to find and work with the source data files.

2. Launch the macro 1: "2. Macro 1 - Collect data" that collects and gathers the data present in the indicated folder in a new Excel workbook named with the "experiment name".

3. Launch the Macro 2: "3. Macro 2 -Analyse data" that processes the data.

\section{b. Calibrate parameters:}


Although RootIX is supplied already calibrated for tomato-seedling root analysis, it is obvious that all plant species have a different root system with different shapes and sizes. Thus, it is possible to personalize this calibration.

1. It is important to adjust the primary and lateral roots discrimination size in cell "E2". Since RootIX assumes that lateral roots are shorter than primary roots, this whole number is used to discriminate between primary and lateral roots. In case the studied plants present shorter primary roots see "special cases" section below.

2. RootlX determines different classes of plants based on two parameters: the number of lateral roots per plant and the size of lateral roots. To get the most suitable ranking, values used to generate it are adjustable in the "Optional" panel:

- Cells C14 to C16 determine the classes of root sizes (smaller size, middle size and large sizes). While the classes are automatically defined based on the discrimination size, they can be modified manually.

- Cells $\mathrm{C} 18$ to $\mathrm{C} 20$ determine the classes of lateral-root number per plant.

\section{c. Several types of calculation:}

RootIX collects and analyzes data from the folder whose path is filled in the worksheet (cell B1). Thus, it is required to store the files to compare in a same folder to make a comparison analysis.

The macro extracts, deduces and calculates several types of information from the data files and it presents them in up to six tables (named Table 1, Table 2, ..., Table 6) that are disposed in a new sheet called "Analyse". The following data are grouped in Table 1 located in the "Analyse" sheet:

- Number of primary and lateral roots per condition

- Different classes of number of lateral roots per plant

- Different classes of lateral root length per plant

- Total primary root lengths per condition (cm)

- Total lateral root lengths per condition (cm)

- Total primary root + lateral root lengths per condition $(\mathrm{cm})$

- Average total primary root + lateral root lengths per plant (cm)

- Average primary root length

- Average lateral root length

- Number of plants with tertiary root

- Average number of tertiary roots per plant.

When samples were measured at two different time points, RootIX calculates the percentage of increase of different parameters between the two time points in Table 2 below the previous one. Five parameters are evaluated:

$1^{\text {st }}$, it indicates the elongation rate of the whole root system (primary and lateral roots taken together).

$2^{\text {nd }}$, it estimates the part of elongation attributed to the primary root.

$3^{\text {rd }}$, the elongation rate of the primary root is indicated.

$4^{\text {th }}$, the rate of additional lateral roots is calculated.

$5^{\text {th }}$ it calculates the total lateral-root length's increase.

$6^{\text {th }}$ it indicates the changes of ramification if new tertiary roots were formed.

A graph presenting these data is generated below Table 2 .

When several replicates of a same condition are analyzed, average and standard deviation of these replicates are calculated for each condition in Table 3 below the second one. In this case, the graph generated present these averaged data below Table 3.

All the calculations in the "Analyse" sheet are linked to the source cells so the user can easily find how the results are calculated.

Furthermore, RootIX also gathered in the table 4, the cumulated lengths of RI and RII of a same plant, in table 5 the RI of each plant and in table 6 the RII of each plant.

It also offers the possibility to shift these tables in horizontal tables that can be used for ANOVA statistical analyses (i.e.: horizontal disposition required for GraphPad Prism one-way ANOVA analyses).

\section{d. Pool the data of multiple replicates}


If the experiment was conducted several times or if there are several files/replicates for a same condition, it could be really useful to pool all the data of each same condition together (without affecting individual files) with the "Optional 4. Pool data" macro. It combines all the data of the different replicates in a new sheet for each condition and then reconducts the analyses on the pooled data in the sheet named "Analyse Pooled Data".

\section{e. Special cases:}

\section{i. Monocotyledons or indiscernible primary root measurements}

If the analyzed plant is a monocotyledon with a fibrous root system, primary root may not be easy to reach. In that case, the first measured root of the plant may be considered as a primary root and the others as lateral roots. The discrimination size may be set at 0 and each new plant (and so primary root) may be recorded with at least four consecutive records of the measurement (eg. $2.43566 \mathrm{~cm}$ record like this "1) 2.43566; 2) 2.43566; 3) 2.43566 ; 4) 2.43566 " on the result file of Image J). It will trigger the manual determination of the root type.

ii. One time point measurements (no growth calculations)

It may occur to compare root shapes between different conditions but regardless to time or growth evolution. In that case it is only required to complete the "conditions" information (row 7 in the "Parameters" sheet) regardless to the starting and ending time points (rows 9 and 10) that can be empty.

\section{iii. Lateral roots longer than primary roots}

In certain conditions, lateral roots could be longer than primary ones. In this particular case it is required to quadruple the record of a measurement thus RootIX will consider the root as a secondary root.

\section{iv. Most of the roots are $\leq 1 \mathrm{~cm}$ or units lengths need to be modified}

When studied roots are very small, the discrimination size could be smaller than one centimeter however RootIX requires a whole number. Then, the simplest solution is to convert the data in millimeters. A button named "Convert length unit of all data", reachable below the "optional" parameters, launches a macro that convert all the data in the wanted unit. Notice that it also allows to convert the unit of the data from and into all the main length units used in the West (international units ( $\mathrm{m}, \mathrm{dm}, \mathrm{cm}, \mathrm{mm}, \mu \mathrm{m})$, American units ( $\mathrm{yd}, \mathrm{ft}, \mathrm{In})$ ). For example, it can convert inches to $\mathrm{cm}$ or $\mathrm{cm}$ into $\mathrm{mm}$ and vice versa.

\section{Results}

\section{WinRhizo - ImageJ measurements comparison}

As part of a study that use algae extracts as biocontrol solutions, we needed to estimate the effect of them on the tomato root architecture. Before launching the RootIX creation, WinRhizo@ was used to analyze root lengths. However, regarding the nature of the images and the media where roots grown, WinRhizo $\subset$ overestimated the major parts of results we were looking for (number of lateral roots, root lengths) (table 1). Indeed, the number of tips detected by WinRhizo $\odot$ was $175 \% \pm 31 \%$ higher than measures conducted with ImageJ manual measurement (table 1 ). The total length of roots on a plate was lightly overvalued of $30 \pm 10 \%$ (table 1). These differences can be mostly explained by the difficulty of the algorithm to detect the crossing roots, thus a root crossing another one may be counted twice (on both sides of the crossed root).

Since manual ImageJ measurements gave a strongly better view of the real root structure, we choose to pursue the lengths analysis with ImageJ.

\section{Measurement}

First, each ten-day-old plant of a culture plate has been measured and saved in a same file (one data file = one condition/one plate of $19.2 \pm 2.4$ plants). Plates were scanned immediately after treatment (TOh) and four days later (T72h). Three independent experiments were conducted. Roots were measured with ImageJ following the indicated sequence for each plant: primary root (RI) followed by its lateral roots: secondary roots (RII) and tertiary roots (RIII).

Secondly, the large amount of data generated needed to be extracted, sorted and analyzed. RootIX was developed for this purpose.

RootIX was ran and gave a high number of information that were then analyzed with GraphPad Prism6 to determine their statistically reproducibility (Fig2). This analysis was conducted on the sum of the primary root length with its lateral roots' length (RI + RII length).

The first time point presented a satisfying reproducibility with a 95\% confidence intervals (Cls) (Fig2A). The second time point display a somewhat similar reproducibility except for the AlgEx1-8000 condition where the difference between the first (R1) and second (R2) replicates is significant (P: 0,0175) while R1 vs third (R3) replicate and R2 vs R3 are not significantly different ( $P$ respectively: 0,4284 and 0,2524) (Fig2B). Keeping in mind this slight biological variability we will assume the reproducibility of the three experiments. Thus, to get a stronger analysis, data of the three experiments were pooled together with the "Optional 4. Pool data" module (see table 2 to get an overview of the generated data).

\section{Root development}

All roots lengths were compared before and $72 \mathrm{~h}$ after treatment in order to determine how treatments affect root elongation. We evaluated the mean of RI and RII development under the different treatments influence with the standard error of the mean (SEM) as error value. Data were also submitted to Dunnett's multiple comparison test against the water condition.

RI elongation (Fig3A). 
$\mathrm{H} 2 \mathrm{O}$ treated plants presented a RI elongation of $7.98 \pm 0.51 \mathrm{~mm}$ when flg22 treatment slightly reduced the elongation with slight difference to $6.02 \pm 0.58 \mathrm{~mm}$ ( $\mathrm{P}$ value $=0.0360$ ).

AlgEx1 at 2000ppm treatment showed a phenotype strongly close to water with an elongation of $7.73 \pm 0.54 \mathrm{~mm}(\mathrm{P}$ value $=0.9994)$. However, when the dose increased to $6000 \mathrm{ppm}$, elongation decreased significantly to $4.45 \pm 0.78 \mathrm{~mm}$ with a $\mathrm{P}$ value $<0.0001$ and when concentration increased to $8000 \mathrm{ppm}$, elongation fell to $2.28 \pm 0.60 \mathrm{~mm}$ with a P value $<0.0001$. Thus, AlgEx1 treatment presented a clear dose effect on RI elongation.

The AlgEx2 treatment induced a stronger and significant effect. Indeed, at the low concentration, $1000 \mathrm{ppm}$, elongation decreased to $5.03 \pm 0.43 \mathrm{~mm}$ with a P value of 0.0002 . The intermediate and high doses of respectively $4000 \mathrm{ppm}$ and $8000 \mathrm{ppm}$ have extremely affected Rl elongation to $0.31 \pm 0.11 \mathrm{~mm}$ and 0.12 $\pm 0.03 \mathrm{~mm}$ respectively with both $\mathrm{P}$ values $<0.0001$. It seemed that AlgEx2 treatment intermediate and high doses blocked primary root elongation.

RII development (Fig3B, C and Fig4).

RootIX has calculated the sum of all the RII-lengths and the number of additional lateral roots of each treated plant. It allows to evaluate the RII development with the mean \pm SEM of two factors $72 \mathrm{~h}$ after treatment: the evolution of the cumulated RIl's length of a plant, named as Rll's growth (Fig3B) and the number of additional lateral roots per plant (ad.Rlls) (Fig3C).

$\mathrm{H} 2 \mathrm{O}$ treated plants presented a RII growth of $3.76 \pm 0.67 \mathrm{~mm}$ that was mainly supported by existing RIl since there was only $0.27 \pm 0.11$ ad. Rlls. Flg22 treatment induced a small reduction of growth to $2.46 \pm 0.58 \mathrm{~mm}$ without stimulating RII formation with $0.20 \pm 0.09$ ad.RIls, both P value were 0.2979 and 0.9994 respectively.

AlgEx1 effect on lateral root development was tenuous at low and intermediate doses but strong at the high concentration. Indeed, the 2000ppm concentration slightly reduce growth to $1.90 \pm 0.45 \mathrm{~mm}$ with a $P$ value of 0.0511 and slightly reduce the lateral root formation with only $0.13 \pm 0.05$ ad. RIls (P value $=0.9334)$. The 6000ppm concentration effect on RIl development was more tangible with a growth that decreased to $1.44 \pm 0.33 \mathrm{~mm}(P$ value $=0.0069)$ in parallel with an increase of $0.46 \pm 0.10$ ad.Rlls being $72 \%$ higher than control but still not significant ( $P$ value $=0.7395)$. Interestingly the highest dose, $8000 \mathrm{ppm}$, offset the individual RII elongation's decrease by a strong lateral root generation of $0.95 \pm 0.20$ ad.RIls being $252 \%$ higher than control $(\mathrm{P}$ value $=$ $0.0002)$ and thus maintained growth to $2.73 \pm 0.84 \mathrm{~mm}$ giving no significative difference with water treatment $(P$ value $=0.5212)$.

The effect of the AlgEx2 treatment is more noteworthy at all doses. The RIl's growth was significantly reduced at the three concentrations (1000, 4000 and $8000 \mathrm{ppm}$ ) respectively $1.061 \pm 0.28 \mathrm{~mm}$ (P value $=0.0009), 1.18 \pm 0.24 \mathrm{~mm}$ (P value 0.0015$), 0.93 \pm 0.15 \mathrm{~mm}$ (Pvalue $=0.0002)$. In the other hand, the lowest dose did not affect significantly the number of ad.Rlls, with a mean of $0.11 \pm 0.05$ per plant ( $P$ value $=0.8587)$. However, intermediate and highest doses stimulate lateral root formation with respectively $0.68 \pm 0.12$ ( $P$ value $=0.0601$ ), and $0.78 \pm 0.11(P$ value $=0.0073$ ) ad. $\mathrm{Rlls}$ per plant being 154 and $191 \%$ much higher than the water condition. It is noteworthy that the high lateral root formation did not counterbalance the RIl's elongation shutdown to maintain a cumulated RII's growth close to the control condition (Fig3B).

Thus, it appeared that both AlgEx1 and AlgEx2 negatively affect RI and RIl elongation in a dose dependent way while they promote lateral root formation at the intermediate and higher doses.

A non-negligible part of this additional lateral roots are adventitious roots that have been formed upon the hypocotyl (Fig3C). Adventitious roots are mostly formed after the AlgEx1 and AlgEx2 treatments and even represent the major part of their additional roots: respectively $84.6 \%$ and $75 \%$ for AlgEx 1 intermediate and high dose and $83.3 \%, 100 \%$ and $94.4 \%$ for AlgEx2 lower, intermediate and high dose. In another hand, AlgEx 1 lower dose triggered only $28.6 \%$ of adventitious roots among the ad.Rlls. This is not so different from the control where the part of adventitious is $18.2 \%$ and $35.7 \%$ for flg 22 and water respectively (Fig3C).

Notice that these proportions of adventitious roots were not calculated by RootIX.

Besides lateral root formation, the size of the RII may also be affected. Indeed, $77.1 \%$ of the tested conditions have a major range of RII comprised between 0.5 and $1 \mathrm{~mm}$ when most of the RII from the other $22.1 \%$ are under $0.5 \mathrm{~mm}$ (Fig4). However, all the conditions allowed the presence of RII longer than $2 \mathrm{~cm} 72 \mathrm{~h}$ after treatment, except AlgEx2 at the higher dose (Fig4).

\section{Discussion}

Root architecture gives an overview of the plant health and ability to colonize its environment. It is mainly represented by root's length and number along with the evolution of these parameters through time. Root architecture may also be conditioned by root diameter and growth direction (Satbhai et al. 2015). RootlX was created to answer quickly this kind of question: "Does the treatment affect root growth and length?".

\section{Advantages}

Our RootIX method does not require to install specific software nor deep configurations. It only needs the two most common biologist software Image J and Excel. To ensure the RootIX operation, measurement must follow this simple sequence: $1^{\text {st }}$ a plant is chosen, $2^{\text {nd }}$ its primary roots (RI) is measured, $3^{\text {rd }}$ its lateral roots (RIls) are measured, $4^{\text {th }}$ a second plant is chosen and so forth. Finally, RootlX provides data in a few clicks: elongation rates; number of additional RIls; RI, RII and total roots (RI+RII of a same plant) sizes (Table 2). Graphs (Fig3 and 4) are ready to be interpreted as well as tables are ready for statistical analysis. For the complete explanation of the tool functioning, please refer to the material and methods chapter. 
This tool is wanted to be totally free and thus enables low-cost experiments that may be useful for researchers with tight budget or who suffers underinvestment such as in emerging and developing countries (UNESCO and Schlegel 2015). Furthermore, RootIX is totally coded with Visual Basic and thus may be upgraded by users to offer new options or to adapt its functionality to unforeseen cases.

\section{Limits}

RootIX was not designed for deep root architecture analysis but to get a rapid and precise overview of a condition effect on the root system focusing on length and number of primary and lateral roots and the evolution of these parameters through time. Since our purpose was to answer to simple question such as "Does a treatment affects root elongation or lateral root formation?", many architecture parameters were not taken in account, such as root diameter, root color or angles. For a deeper and complete architecture analysis, the user may appreciate the free powerful R packages: DART or archiDART (Le Bot et al. 2010; Delory et al. 2018) as well as the ImageJ macro IJ_Rhizo (Pierret et al. 2013).

RootIX offers a fast analysis of data. Nevertheless, the time-consuming part of our analyses was to carry out the measurement manually to avoid errors generated by computers algorithms. Indeed, data obtained with WinRhizo@ were strongly overvalued due to the difficult affiliation of roots that cross each other (Table 2).

However, since it uses ImageJ, the manual measurement could be bypassed, while it is the surest method to avoid artefacts. To this end, it is possible to create/use ImageJ macro that would measure root lengths in the required sequence (RI then RIls for each plant) to allow RootIX analyzes.

It may be frequent to record root length in a large variety of scientific projects but the use of strong software or ImageJ packages such as evocated above are more dedicated to scientists with significant computing backgrounds. For example, IJ_Rhizo is an ImageJ macro and archiDART a R package, both require relevant computing capacities in Image $\mathrm{J}$ macro or $\mathrm{R}$ language to fully perform their operation that may be discouraging for someone with less digital experiences. Finally, soon come the time to choose to invest time in understanding how to use the software, or to simply invest the time on taking the measures and process the data with traditional methods (ruler, ImageJ manual measure, spreadsheet data treatment). Indeed some scientists prefer to use easier methods even more time-consuming (Li et al. 2014; Pereira and Pereira 2018; Fonouni-Farde et al. 2019). RootlX method may be a very good opportunity to fill the gap and bring them an easy to use tool that can help making use of their raw data and save another very time-consuming part of the work.

\section{AlgEx1 and AlgEx2 effect on root system}

In our study, we evaluated the effect of two algal extracts on the root system which are AlgEx1 and AlgEx2. With the help of RootlX we show that AlgEx1 and AlgEx2 negatively affect root elongation while positively favor the formation of lateral root in a dose dependent manner (Fig3 and 4). However, those additional lateral roots were mostly positioned upon the hypocotyls for the medium and high doses of the products. It is known that many endogenous or external aspects may induce the formation of adventitious roots, however it always implicate a complex hormonal modulation where auxin and ethylene seem to play an important role (Li et al. 2009; Pop et al. 2011). These results led us to pursue investigations on root defense elicitation without the higher concentrations. The best compromised would be to increase plant protection against pathogens without affecting plant development.

\section{Conclusion}

RootIX gave a strong and quick access to the essence of simple measurements conducted on roots. Indeed, this tool and method pointed out the dose effect of our treatments on root elongation.

RootIX and this method were developed to give a free to use and easy to reach tool to quickly answer the question of a giving condition effect's on root growth.

Furthermore, since it uses common scientists' tools, ImageJ@ and Excel@, users may be able to improve and adapt RootIX to their own specificities.

\section{Abbreviations}

Flg22: Flagelline 22; RI: Primary root; RII: Secondary root; RIII: Tertiary and other lateral roots; Cl: Confidence interval; ad.RIls: Additional lateral roots

\section{Declarations}

\section{Availability and requirements}

Project name: RootIX

Project home page: none

Operating system(s): Windows 7 or higher

Programming language: Visual Basic

Other requirements: Microsoft Excel 2007 or higher, ImageJ 
License: CC

Any restrictions to use by non-academics: no license needed

\section{Ethics approval and consent to participate}

Not applicable

\section{Consent for publication}

Not applicable

\section{Availability of data and materials}

The datasets used and/or analyzed during the current study are available from the corresponding author on reasonable request.

\section{Competing interests}

The authors declare that they have no competing interests.

\section{Funding}

We gratefully acknowledge support of our research on Root Health and Protection by the ADEME (Agence de l'environnement et de la maîtrise de l'énergie), the University of Rouen, the "Structure fédérative de recherche SFR-Normandie Végétal" from Normandie (France), and the FEDER.

\section{Authors' contributions}

MG managed the experiments, analyzed the plant root images and data, created the RootIX method and tool, wrote the major part of the manuscript. QA and GD prepared and generated the plant cultures, treatments and images. YL participated to the software conception and paper design. AD was a major contributor in writing the paper and supervised the scientific project. MLFG was involved in the data analysis and interpretation. ENO supervised the research project, was involved in the paper organization and corrected the manuscript. MV coordinated the research project, was strongly involved in the paper organization and redaction and corrected the manuscript.

All authors read and approved the final manuscript.

\section{Acknowledgements}

We sincerely acknowledge Caroline Berard for her kind help on statistical analyses.

\section{References}

1. Beck M, Wyrsch I, Strutt J, et al (2014) Expression patterns of FLAGELLIN SENSING 2 map to bacterial entry sites in plant shoots androots. J Exp Bot 65:. https://doi.org/10.1093/jxb/eru366

2. Chuberre C, Plancot B, Driouich A, et al (2018) Plant Immunity Is Compartmentalized and Specialized in Roots. Front Plant Sci 9:. https://doi.org/10.3389/fpls.2018.01692

3. Delory BM, Li M, Topp CN, Lobet G (2018) archiDART v3.0: A new data analysis pipeline allowing the topological analysis of plant root systems. F1000Research 7:22. https://doi.org/10.12688/f1000research.13541.1

4. Dubrovsky JG, Sauer M, Napsucialy-Mendivil S, et al (2008) Auxin acts as a local morphogenetic trigger to specify lateral root founder cells. Proc Natl Acad Sci U S A 105:8790-8794. https://doi.org/10.1073/pnas.0712307105

5. Fonouni-Farde C, Miassod A, Laffont C, et al (2019) Gibberellins negatively regulate the development of Medicago truncatula root system. Sci Rep 9:1-9. https://doi.org/10.1038/s41598-019-38876-1

6. Gou J, Strauss SH, Tsai CJ, et al (2010) Gibberellins Regulate Lateral Root Formation in Populus through Interactions with Auxin and Other Hormones[C] [W]. Plant Cell 22:623-639. https://doi.org/10.1105/tpc.109.073239

7. Jung JKHM, McCouch SRM (2013) Getting to the roots of it: Genetic and hormonal control of root architecture. Front Plant Sci 4:. https://doi.org/10.3389/fpls.2013.00186

8. Le Bot J, Serra V, Fabre J, et al (2010) DART: a software to analyse root system architecture and development from captured images. Plant Soil 326:261273. https://doi.org/10.1007/s11104-009-0005-2

9. Li S-W, Xue L, Xu S, et al (2009) Mediators, Genes and Signaling in Adventitious Rooting. Bot Rev 75:230-247. https://doi.org/10.1007/s12229-009-90299

10. Li W, Duan H, Chen F, et al (2014) Identification of Quantitative Trait Loci Controlling High Calcium Response in Arabidopsis thaliana. PLOS ONE 9:e112511. https://doi.org/10.1371/journal.pone.0112511

11. Millet YA, Danna CH, Clay NK, et al (2010) Innate Immune Responses Activated in Arabidopsis Roots by Microbe-Associated Molecular Patterns. Plant Cell 22:973-990. https://doi.org/10.1105/tpc.109.069658 
12. Pereira JF, Pereira JF (2018) Initial root length in wheat is highly correlated with acid soil tolerance in the field. Sci Agric 75:79-83. https://doi.org/10.1590/1678-992x-2016-0422

13. Pierret A, Gonkhamdee S, Jourdan C, Maeght J-L (2013) IJ_Rhizo: an open-source software to measure scanned images of root samples. Plant Soil 373:531-539. https://doi.org/10.1007/s11104-013-1795-9

14. Plancot B, Santaella C, Jaber R, et al (2013) Deciphering the responses of root border-like cells of Arabidopsis and flax to pathogen-derived elicitors. Plant Physiol 163:1584-1597. https://doi.org/10.1104/pp.113.222356

15. Pop TI, Pamfil D, Bellini C (2011) Auxin Control in the Formation of Adventitious Roots. Not Bot Horti Agrobot Cluj-Napoca 39:307. https://doi.org/10.15835/nbha3916101

16. Robbins NE, Dinneny JR (2015) The divining root: moisture-driven responses of roots at the micro- and macro-scale. J Exp Bot 66:2145-2154. https://doi.org/10.1093/jxb/eru496

17. Rutten JP, ten Tusscher K (2019) In Silico Roots: Room for Growth. Trends Plant Sci 24:250-262. https://doi.org/10.1016/j.tplants.2018.11.005

18. Satbhai SB, Ristova D, Busch W (2015) Underground tuning: quantitative regulation of root growth. J Exp Bot 66:1099-1112. https://doi.org/10.1093/jxb/eru529

19. Schindelin J, Rueden CT, Hiner MC, Eliceiri KW (2015) The ImageJ ecosystem: An open platform for biomedical image analysis: T HE I MAGEJ E COSYSTEM. Mol Reprod Dev 82:518-529. https://doi.org/10.1002/mrd.22489

20. Stringlis IA, Proietti S, Hickman R, et al (2018) Root transcriptional dynamics induced by beneficial rhizobacteria and microbial immune elicitors reveal signatures of adaptation to mutualists. Plant J 93:166-180. https://doi.org/10.1111/tpj.13741

21. Sun J, Xu Y, Ye S, et al (2009) Arabidopsis ASA1 Is Important for Jasmonate-Mediated Regulation of Auxin Biosynthesis and Transport during Lateral Root Formation. Plant Cell 21:1495-1511. https://doi.org/10.1105/tpc.108.064303

22. UNESCO, Schlegel F (eds) (2015) UNESCO science report: towards 2030. UNESCO Publ, Paris

23. Xue R, Zhang B (2007) Increased Endogenous Methyl Jasmonate Altered Leaf and Root Development in Transgenic Soybean Plants. J Genet Genomics 34:339-346. https://doi.org/10.1016/S1673-8527(07)60036-8

\section{Tables}

Table 1: Comparison between WinRhizo@ and ImageJ measurements, respectively automatically and manually conducted on the same images, based on two indicators: number of tips and total root length $(\mathrm{cm})$.

\begin{tabular}{|c|c|c|c|c|c|c|c|}
\hline \multirow[b]{2}{*}{ Condition } & \multirow[b]{2}{*}{ n plants } & \multicolumn{2}{|l|}{ WinRhizo@ } & \multicolumn{2}{|l|}{ ImageJ } & \multicolumn{2}{|c|}{$\begin{array}{l}\text { \% difference: } \\
\text { WinRhizo@ vs ImageJ }\end{array}$} \\
\hline & & Tips & Total length (cm) & Tips & Total length $(\mathrm{cm})$ & Tips & Total length \\
\hline AlgEx1-2000-1 & 20 & 177 & 162.4 & 59 & 142.9 & $+200 \%$ & $+14 \%$ \\
\hline AlgEx1-6000-1 & 19 & 199 & 175.4 & 73 & 141.2 & $+173 \%$ & $+24 \%$ \\
\hline AlgEx1-7200-1 & 17 & 143 & 168.8 & 66 & 124.4 & $+117 \%$ & $+36 \%$ \\
\hline AlgEx1-2000-2 & 18 & 161 & 171.8 & 54 & 127.6 & $+198 \%$ & $+35 \%$ \\
\hline AlgEx1-6000-2 & 17 & 186 & 181.0 & 63 & 127.2 & +195\% & $+42 \%$ \\
\hline AlgEx1-7200-2 & 14 & 156 & 137.7 & 58 & 104.2 & $+169 \%$ & $+32 \%$ \\
\hline Mean \pm SD & $17.5 \pm 2.1$ & $170.3 \pm 20.7$ & $166.2 \pm 15.3$ & $62.2 \pm 6.7$ & $127.9 \pm 14$ & $+175.3 \pm 31.5 \%$ & $+30.5 \pm 10 \%$ \\
\hline
\end{tabular}

Table 2: Overview of the generated data that indicates roots information (lengths and numbers) in the "Table 1" located in the "Analyse" sheet. 


\begin{tabular}{|c|c|c|c|c|c|c|c|c|c|c|c|c|c|c|c|c|c|c|c|c|}
\hline \multirow[t]{2}{*}{ Condition } & \multirow[t]{2}{*}{$\begin{array}{l}\text { Time } \\
\text { Point }\end{array}$} & \multicolumn{2}{|c|}{$\begin{array}{l}\mathrm{nb} / \\
\text { condition }\end{array}$} & \multicolumn{2}{|c|}{$\begin{array}{l}\mathrm{nb} \text { of } \\
\text { RII/plant }\end{array}$} & \multicolumn{4}{|c|}{$\begin{array}{l}\text { number of plant } \\
\text { with }\end{array}$} & \multicolumn{4}{|c|}{ number RII } & \multicolumn{3}{|c|}{$\begin{array}{l}\text { total lengths / } \\
\text { condition }(\mathrm{cm})\end{array}$} & \multicolumn{3}{|c|}{$\begin{array}{l}\text { mean lengths / } \\
\text { plant }(\mathrm{cm})\end{array}$} & \multirow{2}{*}{$\begin{array}{l}\text { std } \\
\text { lens } \\
\text { (cm } \\
\text { RI }\end{array}$} \\
\hline & & RI & RII & mean & $\begin{array}{l}\text { std } \\
\text { dev }\end{array}$ & $\begin{array}{l}<= \\
2 \\
\text { RII }\end{array}$ & $\begin{array}{l}> \\
2 \\
=< \\
4 \\
\text { RII }\end{array}$ & $\begin{array}{l}> \\
4 \\
=< \\
6 \\
\text { RII }\end{array}$ & $\begin{array}{l}> \\
6 \\
\text { RII }\end{array}$ & $\begin{array}{l}< \\
0.5 \\
\mathrm{~cm}\end{array}$ & $\begin{array}{l}>= \\
0.5 \\
<1 \\
\mathrm{~cm}\end{array}$ & $\begin{array}{l}>= \\
1< \\
2 \\
\mathrm{~cm}\end{array}$ & $\begin{array}{l}>= \\
2 \\
\mathrm{~cm}\end{array}$ & Rls & Rlls & $\begin{array}{l}\text { Rls + } \\
\text { Rlls }\end{array}$ & $\begin{array}{l}\text { Rls } \\
+ \\
\text { Rlls }\end{array}$ & RI & $\begin{array}{l}\text { sum } \\
\text { of } \\
\text { RII }\end{array}$ & \\
\hline All3-2000 & TOh & 54 & 218 & 4.0 & 2.09 & 11 & 24 & 13 & 6 & 84 & 105 & 29 & 0 & 373.5 & 140.7 & 514.2 & 9.5 & 6.9 & 2.6 & 1.1 \\
\hline All3-2000 & T72h & 54 & 220 & 4.1 & 2.1 & 11 & 24 & 13 & 6 & 73 & 116 & 28 & 3 & 415.2 & 149.9 & 565.1 & 10.5 & 7.7 & 2.8 & 1.2 \\
\hline All3-6000 & TOh & 56 & 196 & 3.5 & 1.63 & 17 & 25 & 11 & 3 & 58 & 98 & 39 & 1 & 375.9 & 141.1 & 517.0 & 9.2 & 6.7 & 2.5 & 0.7 \\
\hline All3-6000 & $\mathrm{T} 72 \mathrm{~h}$ & 56 & 220 & 3.9 & 1.76 & 10 & 29 & 11 & 6 & 79 & 99 & 41 & 1 & 399.9 & 148.0 & 547.9 & 9.8 & 7.1 & 2.6 & 1.0 \\
\hline All3-8000 & TOh & 59 & 200 & 3.4 & 2.06 & 23 & 15 & 19 & 2 & 63 & 103 & 33 & 1 & 400.9 & 137.5 & 538.4 & 9.1 & 6.8 & 2.3 & 1.1 \\
\hline All3-8000 & $\mathrm{T} 72 \mathrm{~h}$ & 59 & 255 & 4.3 & 2.47 & 13 & 20 & 16 & 10 & 113 & 107 & 35 & 0 & 413.9 & 151.4 & 565.3 & 9.6 & 7.0 & 2.6 & 1.1 \\
\hline $\begin{array}{l}\text { All45B- } \\
1000\end{array}$ & TOh & 57 & 195 & 3.4 & 2.39 & 22 & 21 & 9 & 5 & 75 & 86 & 32 & 2 & 390.3 & 135.0 & 525.3 & 9.2 & 6.8 & 2.4 & 1.0 \\
\hline $\begin{array}{l}\text { All45B- } \\
1000\end{array}$ & T72h & 57 & 200 & 3.5 & 2.32 & 22 & 19 & 11 & 5 & 77 & 89 & 29 & 5 & 418.8 & 140.5 & 559.3 & 9.8 & 7.3 & 2.5 & 1.1 \\
\hline $\begin{array}{l}\text { All45B- } \\
4000\end{array}$ & TOh & 60 & 190 & 3.2 & 2.14 & 24 & 23 & 10 & 3 & 47 & 104 & 37 & 2 & 389.9 & 138.3 & 528.2 & 8.8 & 6.5 & 2.3 & 1.0 \\
\hline $\begin{array}{l}\text { All45B- } \\
4000\end{array}$ & $\mathrm{~T} 72 \mathrm{~h}$ & 60 & 230 & 3.8 & 2.32 & 18 & 21 & 15 & 6 & 90 & 101 & 36 & 3 & 391.4 & 144.8 & 536.2 & 8.9 & 6.5 & 2.4 & 1.1 \\
\hline $\begin{array}{l}\text { All45B- } \\
8000\end{array}$ & TOh & 69 & 261 & 3.8 & 2.11 & 21 & 25 & 18 & 5 & 79 & 135 & 46 & 1 & 458.9 & 183.4 & 642.3 & 9.3 & 6.6 & 2.7 & 1.1 \\
\hline $\begin{array}{l}\text { All45B- } \\
8000\end{array}$ & T72h & 69 & 312 & 4.5 & 2.45 & 15 & 21 & 20 & 13 & 128 & 142 & 41 & 1 & 458.7 & 187.4 & 646.1 & 9.4 & 6.6 & 2.7 & 1.0 \\
\hline flg22 & TOh & 54 & 170 & 3.1 & 1.96 & 21 & 23 & 6 & 4 & 55 & 93 & 22 & 0 & 359.6 & 111.8 & 471.4 & 8.7 & 6.7 & 2.1 & 1.0 \\
\hline flg22 & T72h & 54 & 180 & 3.3 & 1.97 & 19 & 22 & 8 & 5 & 63 & 87 & 27 & 3 & 392.0 & 124.3 & 516.3 & 9.6 & 7.3 & 2.3 & 1.1 \\
\hline $\mathrm{H} 2 \mathrm{O}$ & TOh & 52 & 175 & 3.4 & 1.89 & 20 & 19 & 10 & 3 & 63 & 88 & 24 & 0 & 344.5 & 112.3 & 456.8 & 8.8 & 6.6 & 2.2 & 1.0 \\
\hline $\mathrm{H} 2 \mathrm{O}$ & $\mathrm{T} 72 \mathrm{~h}$ & 52 & 187 & 3.6 & 2.02 & 19 & 17 & 12 & 4 & 66 & 83 & 37 & 1 & 386.0 & 131.4 & 517.5 & 10.0 & 7.4 & 2.5 & 1.1 \\
\hline
\end{tabular}

\section{Figures}




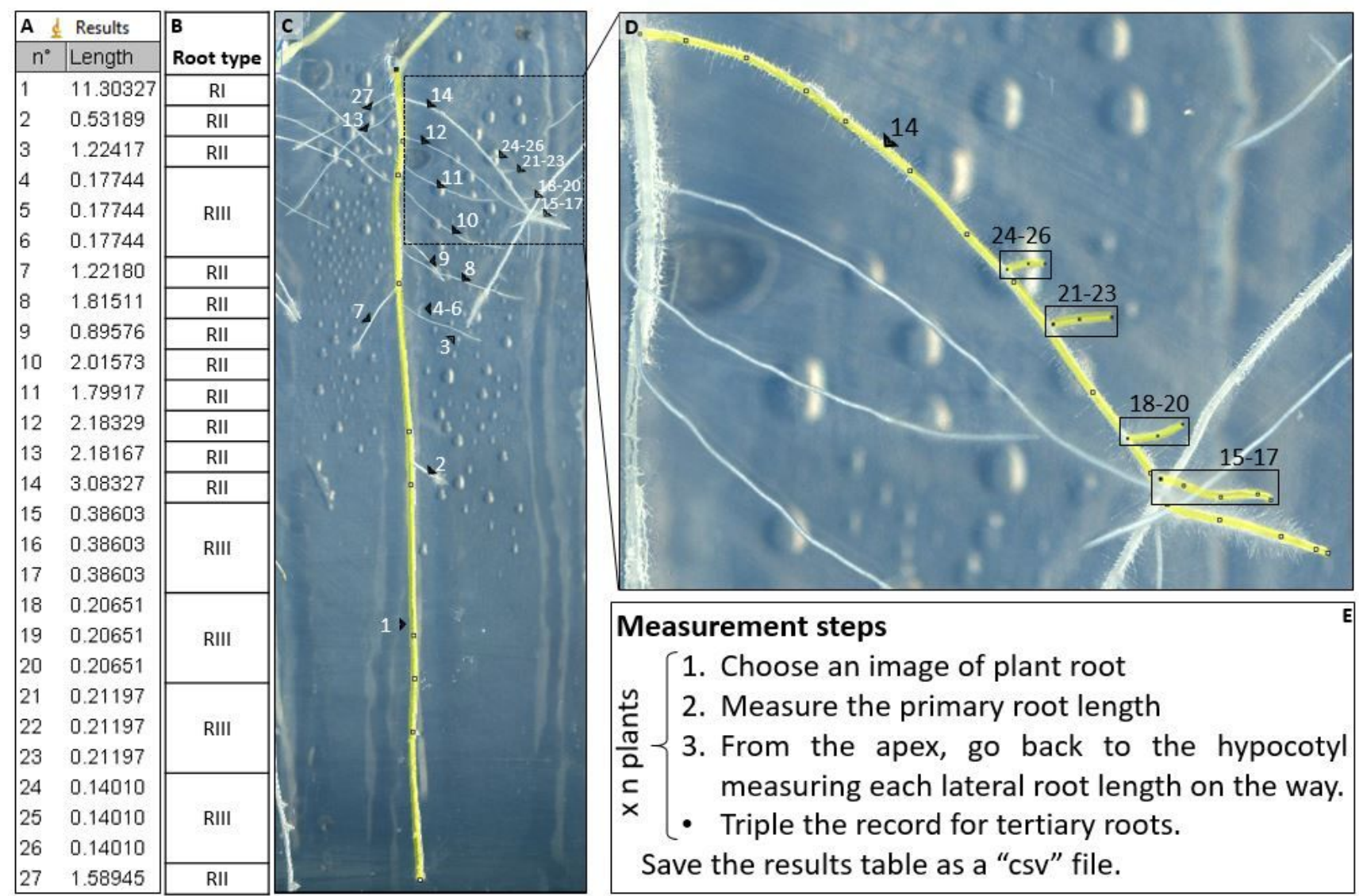

Figure 1

Required measurement steps. Each plant is measured following a simple sequence of steps that allows RootlX to identify the different root types. A. Table of results provided after measurements with ImageJ. First column indicates the number of the record, second column the length. B. Table of the different root types (primary root (RI), secondary root (RII), tertiary root (RIII)) corresponding to the records of the results table that will be deduced by RootIX. C. Image of the measured plant with the number of each recorded root in the table of results. The RI appears in yellow. D. Zoom of the dashed frame where RIII are identified on a RII. E. Explanation of the measurement steps. The number of plants and the corresponding Rl, are not limited in the ImageJ results table. This results table will be saved as a "csv" file. One file must be related to one condition, one time point and n plants

A $95 \%$ Confidence Intervals (Tukey)TOh

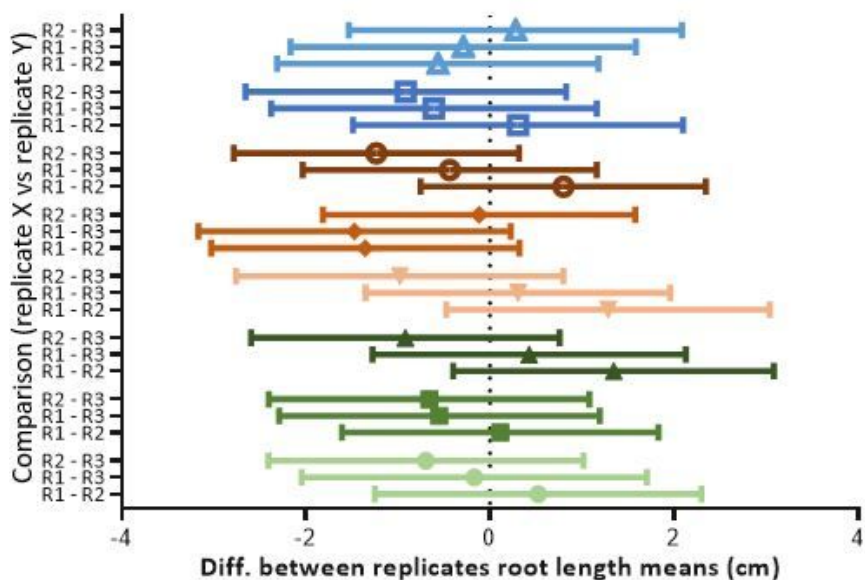

Diff. between replicates root length means $(\mathrm{cm})$
B $\quad 95 \%$ Confidence Intervals (Tukey) $\mathrm{T72h}$

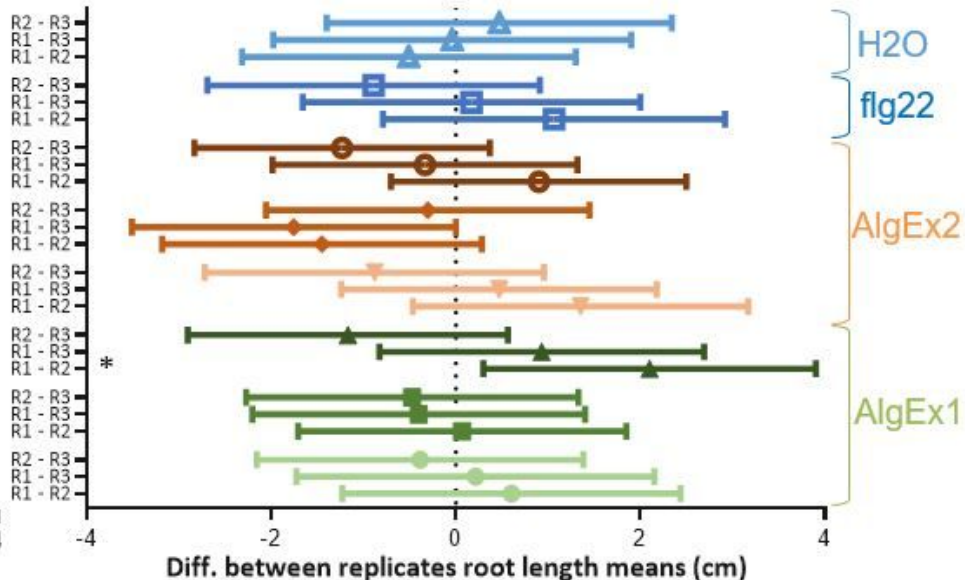

Diff. between replicates root length means $(\mathrm{cm})$

Figure 2

Differences between replicates root length means before treatment (A) and $72 \mathrm{~h}$ post treatment (B). Each difference is represented as a central dote with $95 \%$ confidence intervals. Sum of each primary root length with their lateral roots' length (RI + RII length) was calculated by RootIX and provided in the "Table 4" of the "Analyse" sheet. All replicates from this table were compared with each other using GraphPad Prism 6.0 Tukey's multiple comparison test to evaluate their 
reproducibility (P values: no symbol $>0,05 ; *<0,05)$. R1, R2, R3 are respectively the first, the second and the third replicate. The treatment evaluated are water

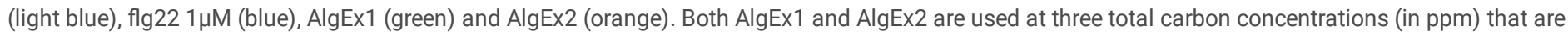
represented with light colors (2000 and 1000 ppm respectively) medium colors (6000 and 4000 ppm respectively) and dark colors (8000 ppm for both).

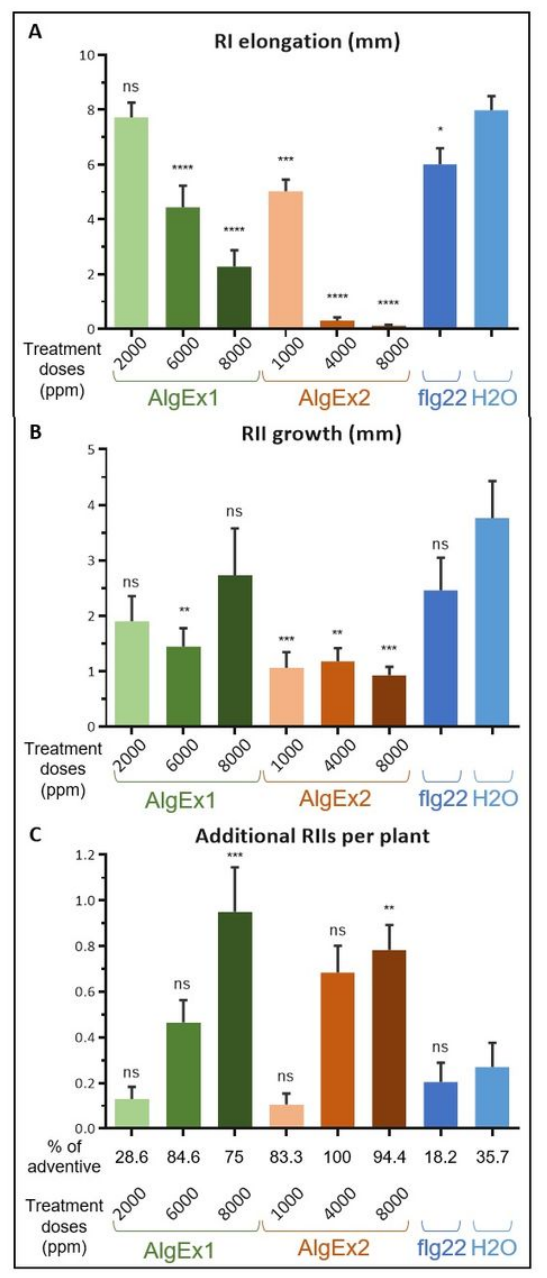

Figure 3

RootIX graphics presenting the root development variations three days after treatment. Plant were treated with the AlgEx 1 treatment (green) with three carbon concentrations (in ppm): 2000, 6000, 8000; or with the AlgEx2 treatment (brown) with three carbon concentrations (in ppm): 1000, 4000, 8000; or with two control solution: water (light blue) and flg22 (dark blue). A) Elongation mean of the primary root (RI). B) Growth mean of the lateral roots (RII) cumulated lengths. C) Average number of additional Rlls per plant. The percentage of the adventitious RII formed upon the hypocotyl among the total lateral roots formed after treatment is indicated below the graph. Error bars: standard error of the mean. $\mathrm{N}=57.6 \pm 5.1$ plants for each condition. Statistical analyses were Dunnett multiple comparison tests against water $(\mathrm{a}=0,05)$. P values: $\mathrm{ns}>0,05 ; *<0,05 ; * \star<0,01 ; * \star *<0,001 ; * \star \star *<0,0001$; 


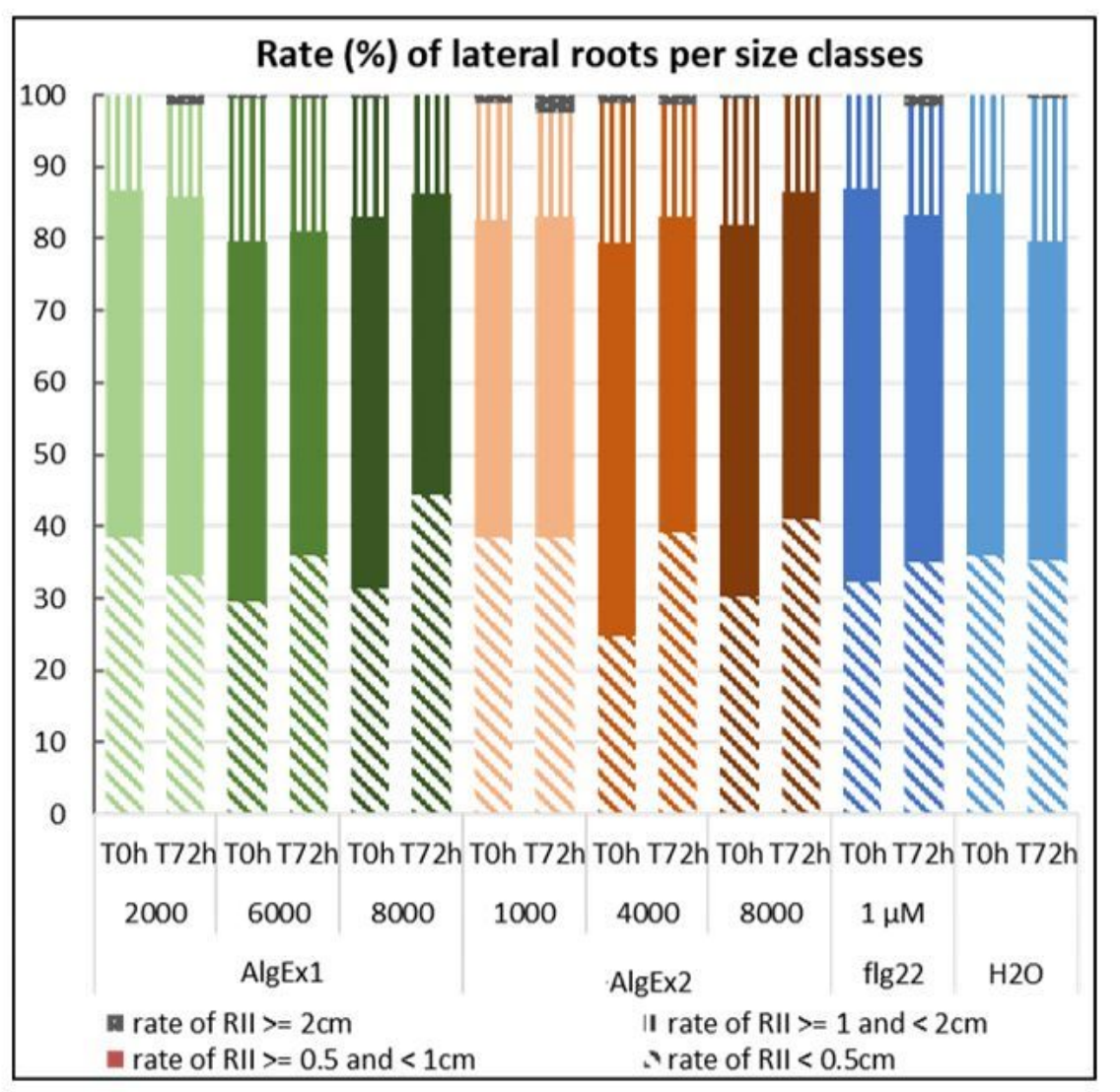

Figure 4

RootIX analyses indicate that lateral roots (RIIs) are affected by different treatments. RootlX graphic evaluating the lateral root distribution into four size ranges: $<0.5 \mathrm{~cm}$ (diagonal hatched bars), $>=0.5<1 \mathrm{~cm}$ (full bars), $>=1<2 \mathrm{~cm}$ (vertical hatched bars), $>2 \mathrm{~cm}$ (checked pattern bars). $\mathrm{N}=57.6 \pm 5.1$ plants for each condition. 